\title{
Potential Regulators of Sporadic ALS Development and Alternative Therapeutic Options
}

\author{
Morenci M. Manning, Marie Kelly-Worden \\ Ball State University, Muncie, USA \\ Email: mlkellyworde@bsu.edu \\ Received 13 February 2015; accepted 28 February 2015; published 5 March 2015 \\ Copyright (C) 2015 by authors and Scientific Research Publishing Inc. \\ This work is licensed under the Creative Commons Attribution International License (CC BY). \\ http://creativecommons.org/licenses/by/4.0/

(c) (i) Open Access

\begin{abstract}
Amyotrophic Lateral Sclerosis (ALS) is the most common neurodegenerative disorder. It is also among the most lethal as life expectancy is between 2 and 5 years after diagnosis. Sporadic ALS (sALS) makes up $\mathbf{9 0 \%}$ of all ALS cases with little known about the exact mechanism of pathogenesis. Many potential regulators of sALS development have been proposed, several of which are examined in this review with supporting evidence. Interplay among these factors is examined more closely in hopes of shedding more light on sALS pathophysiology. There is a paucity of effective treatment options as the only FDA-approved drug for use, riluzole, has a positive but modest benefit in improving survival. Other treatments available merely target ameliorating symptoms of ALS. Alternative treatment options are also discussed. This study ultimately aims to make relevant connections among factors implicated in SALS development and to highlight alternative forms of treatment in improving neuron function and status, albeit controversial.
\end{abstract}

\section{Keywords}

Amyotrophic Lateral Sclerosis, ALS, Sporadic ALS, Neurodegeneration

\section{Introduction}

\subsection{What Is ALS?}

Amyotrophic Lateral Sclerosis (ALS) is a progressive neurodegenerative disease affecting both upper and lower motor neurons in the motor cortex, brain stem, and spinal cord [1]. Damage to the motor neurons rapidly leads to muscle weakness, subsequent deterioration of function, and paralysis. Additionally, the individuals affected live no longer than 2 - 5 years after diagnosis on average [2] [3]. Some individuals live 10 years and even fewer 
live beyond 20 years. Equally as dismal as the survival rate for ALS is the quality of life for those diagnosed. As muscles lose essential connections to the brain's commands, vital tasks for survival such as moving autonomously, chewing, and swallowing become cumbersome and eventually impossible. Recent enlightenment into mitochondrial defects associated with ALS has allowed the facilitation of dietary modifications in slowing the progression of ALS. These efforts, although successful, have not provided a large enough sample to support the claim [4]. Unfortunately, rapid disease progression outpaces and overshadows the benefit to date.

\subsection{Incidence and Demographic of ALS}

The incidence of ALS in the US population is approximately 2.6 cases per 100,000 [1]. The demographic of those predominantly affected by ALS are Caucasian males between 60 and 69 years of age [3]. However, onset of the disease can arise within an individual as early as in their twenties.

\subsection{Etiology of ALS}

The etiology of ALS has yet to be elucidated, but it is widely accepted that multiple intersecting factors contribute to its development. Factors such as genetics and environment have been implicated in ALS development, among others. ALS is categorized into two primary forms, sporadic and familial. Approximately $90 \%$ of diagnosed cases arise sporadically and the remaining $10 \%$ are of familial origin [5]. Though there is little evidence explaining the rise of sporadic cases, there is a growing understanding of the familial. Of the small percentage of familial cases of ALS, roughly $20 \%$ share a mutation in one of the body's antioxidant sources, superoxide dismutase type 1 gene (SOD1) [6]. Fortunately, this finding has aided in identifying other genetic predispositions shared among those affected. However, much more work needs to be done in order to better target culprit regulators of sporadic ALS development.

\subsection{Purpose of This Investigation}

The purpose of this investigation is to examine more closely the intersecting factors of those affected by sporadic ALS, in hopes of shedding light on possible regulators of its development. Additionally, supplementary treatment options will be investigated. There currently exists no therapy available to slow disease progression [6]. With a better understanding of the common factors among patients and a broader range of potential therapeutic options, novel approaches to treatment can be assessed and pathways of development can be examined more thoroughly.

\section{Implications in Sporadic ALS Development}

Many studies have attempted to take a genetic approach in delineating the cause of sALS, as it has played such an integral role in understanding familial ALS pathogenesis (fALS). However, few genetic factors alone have been found to contribute significantly in sALS. There is more supporting the theory of interplay between genetic predisposition and environmental factors. Mounting evidence suggests a strong correlation between environmental, social, and biological factors with dramatically increased incidence of the sporadic form of the disease. Factors such as glutamate excitotoxicity associated with diet and genetics, oxidative stress, and mitochondrial dysfunction are of significant implications to name a few [1] [7] [8]. Water quality has also been attributed to development of ALS with a 50 - 100 fold increase in sporadic cases within populations where water quality was poor [9] [10]. It is anticipated that the intersection of these factors, and others, will facilitate a better understanding of sALS development.

\subsection{Oxidative Stress and Mitochondrial Dysfunction}

Oxidative stress and mitochondrial dysfunction go hand-in-hand in multiple neurological disorders and even more so in ALS. With the leakage of reactive oxygen species from the mitochondria observed with loss of function, it's no surprise at the strength of the interplay between the two in sALS [11]. Additionally, motor neurons tend to have much longer axons than other neurons constituting the CNS. This increased surface area requires more mitochondria in order to supply enough energy input to generate sufficient action potentials, thus making these neurons more susceptible to mitochondrial mishaps [11]. 
There are several ways to measure oxidative stress. Quantifying 8-OxodG and Isoprotane (IsoP) levels are two ways of facilitating this. Mitsumoto et al. observed elevated levels of both in sALS patients (Table 1). More specifically, they found an approximate $36 \%$ increase in 8 -OxodG in the urine of sALS patients compared to controls $(57 \mathrm{nmol} / \mathrm{mmol}$ vs. $42 \mathrm{nmol} / \mathrm{mmol}$ respectively). Also, there was a $60 \%$ increase in IsoP levels of sALS patients compared to controls $(0.8 \mathrm{nmol} / \mathrm{mmol}$ vs. $0.5 \mathrm{nmol} / \mathrm{mmol})$ [8].

As mentioned, SOD1 is an important regulator of fALS development and has been shown to play a role in sALS pathogenesis as well. Mutations in the SOD1 gene comprise a number of sALS cases. The mitochondria houses between $1 \%$ and $5 \%$ of SOD1, and mitochondrial abnormalities are observed prior to when the ALS phenotype fully manifests [11]. Defects in this gene are often the premiere highlight when dissecting pathophysiology, with few other solid leads, and rightfully so. Mutations in this gene have accounted for roughly $25 \%$ of familial cases and roughly $4 \%$ of sporadic cases [12]. However, an additional molecule of interest should also be added to the conversation, Coenzyme $\mathrm{Q}_{10}(\mathrm{CoQ} 10)$. Its role in mitochondrial function and plasma levels in response to induction of ALS suggest a culprit role in etiology of sporadic ALS [11].

CoQ10 is a quintessential cofactor in cellular respiration; more specifically, it is associated with oxidative phosphorylation (Table 1). It also functions as an antioxidant serving to neutralize potential oxygen radicals. Fibroblast CoQ10 deficient cultures display decreased activities of complex II + III, complex III, and complex IV, as well as reduced expression of mitochondrial oxidative phosphorylation proteins, decreased mitochondrial membrane potential, activation of mitochondrial permeability transition, and increased production of reactive oxygen species (ROS) along with reduced growth rates [13]. Its protective effects from oxidative damage and role in mitochondrial function, coupled to decreased plasma concentration with mitochondrial insult, make CoQ10 a prime target for mitochondrial function assessment in sporadic ALS [11].

Both $\mathrm{CoQ} 10$ and 8-OHdG can be used as markers of mitochondrial dysfunction. Oxidized CoQ10 levels have been measured in the cerebrospinal fluid (CSF) of sALS patients and controls and found to be approximately $85.7 \%$ and $68.2 \%$ respectively. $8-\mathrm{OHdG}$ levels were also found to be increased by $59 \%$ in the CSF of sALS patients compared to controls $(5.1 \mathrm{pg} / \mathrm{ml}$ vs. $1.7 \mathrm{pg} / \mathrm{ml}$ respectively) [11].

\subsection{Glutamate Excitotoxicity}

Glutamate excitotoxicity is a chief characteristic of ALS. It causes overstimulation of neurons by excess glutamate in the synaptic cleft and yields the characteristic deformations in neurons ultimately leading to their cell death [14]. This appears to be catalyzed by increased permeability of calcium by NMDA and AMPA receptors, leading to excess intracellular calcium [14] [15]. This abundance of intracellular calcium has been demonstrated to be directly linked to mitochondrial dysfunction via nitric oxide (NO) stimulation, further illuminating the interplay between multiple factors in disease development [16]. Glutamate levels were found to be increased in the CSF of $40 \%$ of 400 patients with sporadic ALS, with a strong correlation between glutamate levels and disease progression [17]. Thus, glutamate excitotoxicity still serves as the best hypothesized etiologic factor. This is further supported by the fact that Riluzole owes its success to reducing this prominent feature of ALS pathology [18].

\subsection{Other Genetic Factors}

More recent developments have identified a key feature of sporadic ALS pathology, TDP-43 displacement from

Table 1. Possible causes, factors and observations of ALS.

\begin{tabular}{|c|c|c|}
\hline Possible Causes of ALS & Factors Examined & sALS vs. controls \\
\hline \multirow{4}{*}{ Mitochondrial dysfunction } & Urinary 8-OxodG & $\uparrow 8$-OxodG \\
\hline & Urinary $15-F_{2 t}$ isoprostane (IsoP) & $\uparrow \mathrm{IsoP}$ \\
\hline & Oxidized CoQ10 levels in CSF & $\uparrow$ Oxidized CoQ10 \\
\hline & 8-OHdG levels in CSF & $\uparrow 8-\mathrm{OHdG}$ \\
\hline Glutamate toxicity & Glutamate levels in CSF & $\uparrow$ Glutamate \\
\hline \multirow{2}{*}{ Toxin exposure } & Cigarette smoking & $\uparrow \mathrm{ALS}$ risk \\
\hline & Cyanobacteria (BMAA levels) & $\uparrow \mathrm{BMAA}$ (not found in controls) \\
\hline Genetic mutations & TDP-43 TARBDP & $\uparrow$ Missense mutations \\
\hline
\end{tabular}


the nucleus and subsequent increases of inclusion bodies in the cytoplasm [19]. Transactive response DNA binding protein 43, TDP-43, as its name implies is a DNA binding protein with a vital role in both familial and sporadic ALS [20]. Mutations in this gene are associated with neurodegenerative diseases usually involving the frontotemporal lobe [21]. TDP-43 abnormality has been demonstrated to be caused by increased calcium permeability by AMPA receptors [22]. Of 9 individuals examined with sALS, 8 of them possessed missense mutations in TDP-43 [23].

\subsection{Toxin Exposure}

Many toxins have been investigated in order to dissect their respective roles in ALS including pesticides and insecticides, tobacco smoke, heavy metals including: mercury, selenium, zinc, and even lead [24]. One source of toxins, noteworthy of mention, is tobacco smoke; as it is an environmental factor largely controlled by the individual. Cigarette smoke has been consistently shown to increase chances of developing ALS [24]. Approximately $57.3 \%$ of sALS patients reported smoking compared to $41.7 \%$ of controls [25].

Finally, another toxin of importance is BMAA ( $\beta$-N-methylamino-L-alanine), which is a toxin derived from cyanobacteria. Multiple studies have demonstrated strong correlations between ALS clusters and exposure to this cyanobacterial neurotoxin [9]. In fact, in one particular study, it was detected in high concentrations in all ALS cases examined but no controls [25]. Bradley and Mash (2009) measured levels of BMAA in 13 sALS patients and 12 controls. They detected an average of $134 \mathrm{ug} / \mathrm{g}$ in ALS patients, but detected no BMAA in controls. This poisonous amino acid has been reported to be the culprit of ALS spikes in both the Kii Peninsula in Japan and on the island of Guam [26]. Studies have consistently shown that poor water sources and food (particularly fish and cycad seeds) are in sufficient BMAA concentrations to cause neurological dysfunction such as is observed in ALS [26].

\section{Treatment}

ALS is difficult to treat primarily as a result of the lag between disease manifestations and actual diagnosis, and the fact that the etiology remains unknown [27]. Damage incurs in the nervous system before the person succumbs to the loss of motor function and subsequent mobility. Our investigation sought to highlight alternative treatment options not yet tested in humans. In this study, one currently used treatment for ALS and two other potential therapeutic options are being further examined. The first, Riluzole, a drug approved by the FDA in 1996 to treat ALS, one in early clinical trial stages (HUCBCs), and the other not yet approved for general use in humans, AAV9-ADAR2 (Table 2).

\subsection{Riluzole}

As of yet, only one therapeutic drug has demonstrated sufficient capacity to prolong survival in humans with

Table 2. Comparison of Available Therapy with Therapeutic Candidates in ALS Treatment.

\begin{tabular}{|c|c|c|c|c|}
\hline Substance & Subject & Benefit & $\begin{array}{l}\text { Adverse Effects } \\
\text { Observed }\end{array}$ & Drawbacks \\
\hline AAV9-ADAR2 injection & Mice & $\begin{array}{l}\text { - Rescue of motor neurons } \\
\text { from death } \\
\text { - Neuron regeneration }\end{array}$ & N/A & $\begin{array}{l}\text { - Little known } \\
\text { mechanism }\end{array}$ \\
\hline Riluzole & Humans & $\begin{array}{l}\text { - Prolong overall survival?/delay } \\
\text { disease progression }\end{array}$ & $\begin{array}{ll}\text { - } & \text { Diarrhea } \\
\text { - } & \text { Dizziness } \\
\text { - } & \text { Fatigue } \\
\text { - } & \text { Nausea }\end{array}$ & \\
\hline $\begin{array}{l}\text { Stem cells (Human } \\
\text { Umbilical Cord Blood } \\
\text { Cells, HUCBCs) }\end{array}$ & Mice & $\begin{array}{l}\text { - } \text { Low immunogenicity } \\
\text { - } \text { Retard disease progression } \\
\text { - } \quad \text { Improlong survival } \\
\text { - } \quad \text { attenuromuscular transmission } \\
\text { astrogliosis }\end{array}$ & $\mathrm{N} / \mathrm{A}$ & $\begin{array}{l}\text { - Ethical issue } \\
\text { with use }\end{array}$ \\
\hline
\end{tabular}


ALS (Riluzole [18]). Riluzole functions by blocking voltage-gated sodium channels and lowers the inward sodium and calcium current by blocking NMDA receptors responsible for glutamate excitotoxicity [28]. This manipulation of excitotoxicity, a major defining characteristic of sporadic ALS, makes Riluzole somewhat effective at delaying progression of disease. Unfortunately, this drug only moderately delays disease progression and does not restore motor function, improve quality of life, or reverse damage already incurred. Also, adverse effects have been attributed to administration of this drug such as dizziness, nausea, fatigue, diarrhea, and possibly drowsiness [18].

\subsection{AAV9-ADAR2}

Adenosine deaminase acting on RNA2 (ADAR2) is an enzyme that edits RNA. Low levels of this enzyme have been found to coincide with increased expression of the unedited glutamate/arginine GluA2 region of the AMPA receptor. This has been shown to lead directly to motor neuron death by an increased calcium-AMPA receptor permeability mechanism [22]. This therapeutic option also manipulates a key component of excitotoxicity. Mice brains treated with ADAR2enzymeprotein demonstrated neuron regeneration, rescue of motor neurons from death, and reversal of the characteristic TDP-43 phenotype of classic sporadic ALS motor neurons [22].

\subsection{Stem Cells}

Stem cells, particularly Human Umbilical Cord Blood Cells (HUCBCs), have been used in animal models of ALS and achieved promising results. HUCBCs have the capacity to differentiate into many different types of cells, the most important in this case, being neurons [27]. They have also been shown to secrete growth factors, supporting the microenvironment of healthy motor neurons [27]. This makes HUCBCs a promising therapeutic target in ALS treatment.

One study of particular significance involved injecting SOD1-G93A ALS model mice with HUCBCs. Delayed progression of disease and a modest increase in overall survival were observed. More specifically, they observed a reduction in proinflammatory cytokines in the brain and spinal cord. Although motor function was not explicitly observed in this study, a slight beneficial effect on motor function was observed [29]. This benefit is supported by several other studies performed in mice demonstrating improvement in overall survival, retardation of disease progression, and improving function of existing motor neurons [30]-[32].

\section{Discussion}

\subsection{Glutamate Toxicity as an Initiator}

When examining each of the factors assessed to cause sALS, it is interesting to note that of all the factors, only one is a direct precusor to glutamate excitotoxicity (of those uncovered in this investigation). The factors mentioned have primarily resulted from dysfunctions in one of the other factors listed in Table 1, which are likely to be downstream of glutamate excitotoxicity. For example, mitochondrial dysfunction is directly related to oxidative stress. Only TDP-43 abnormality has been demonstrated to be caused by increased calcium permeability associated with glutamate AMPA receptors.

Glutamate excitotoxicity appears to be the factor of the greatest significance in understanding pathogenesis of sALS. Discovering the predisposition for this toxic effect of excess glutamate on neurons and the underlying intracellular mechanisms responsible seems to be of grave importance in improving functional neuron status in ALS. Also, determining what makes the individual susceptible to these toxic effects appears to be the best approach in determining etiology.

Oxidative stress markers, 8-OxodG and IsoP, were examined in a subset of sALS patients. They were found to be increased in the urine of sALS patients [8]. Similarly, 8-OHdG and CoQ10, markers of mitochondrial dysfunction, were also found to be increased in sALS patients [11]. As mentioned, both malfunctions appear to be consequences of glutamate-induced death of neurons. Glutamate levels were significantly increased in the CSF of sALS patients when compared with controls. Additionally, each of the toxins examined could potentially be key in triggering sALS development; but more work needed to be done using more direct study designs that could form a causative relationship between sALS development and exposure to the poison. Thus, the best question is no longer what causes sALS, but which factor lies upstream in the pathway. 


\subsection{Overview of Culprits and Treatments Presented}

This review of factors potentially responsible for sporadic ALS development has not been comprehensive. It has rather been an attempt to examine the intersection of some of the most prevalent factors and hypothesize the most likely initiator, or the interplay among the most significant. There are other factors with potential roles, but those discussed in this review are of the most significant contributions and the most thoroughly documented. Another aim of this review is to build a case for alternative treatments with the most potential to reverse the damage resulting from sALS, although controversial.

The three treatments discussed in this study are not directly comparable as the test subjects, and parameters are not symmetrical. The ADAR2 protein and the stem cell therapy studies were both conducted in mice, while the Riluzole study was conducted in humans. The purpose of this comparison is rather to emphasize the dearth of treatments in ALS and to highlight alternate potential candidates for novel therapeutic approaches. There are clinical trials currently taking place involving HUCBCs in humans in the treatment of ALS, but the ADAR2 therapy has yet to be tested in humans. The success of each treatment in animal models (Table 2) should be strongly considered when discussing a potential transition to treatment in humans, especially given the limited toxicity.

Although stem cell use has been a topic of controversial debate for the last two decades, HUCBCs remain an option worth further consideration in sporadic ALS treatment given the success observed in the studies mentioned. Unlike traditional embryonic stem cell harvesting, human umbilical cord blood harvesting does not cause harm to the fetus since the umbilical cord is detached after birth. This makes this form of treatment ethical. Also, women now have the option of donating the cord blood, so no one overrides an individual's will to provide these HUCBCs or not. However, one drawback of stem cells is still the lack of knowledge regarding the mechanism of action [27].

What is known is that glutamate excitotoxicity plays a key role in sALS. Glutamate is the chief excitatory neurotransmitter in the central nervous system [33]. As mentioned, so far glutamate-induced toxicity has appeared to be most upstream regulator of sALS development. It has been shown to be induced by injury to the central nervous system [33]. More research needs to be done in order to determine the specific intracellular mechanism. A better understanding of this mechanism may provide a deeper understanding of the etiological regulator of sALS development. It may also, in turn, facilitate more effective treatment strategies.

\section{References}

[1] Anand, A. Thakur, K. and Gupta, P.K. (2013) ALS and Oxidative Stress: The Neurovascular Scenario. Oxidative Medicine and Cellular Longevity, 2013, 1-14.

[2] Brooks, B.R., Sanjak, M., Belden, D., Juhasz-Poscine, K. and Waclawik, A. (2000) In: Brown Jr., R.H., Meininger, V. and Swash, M., Eds, Amyotrophic Lateral Sclerosis, Dunitz, London, 31-58.

[3] Mehta, P., Antao, V., Kaye, W., Sanchez, M., Williamson, D., Bryan, L., Muravov, O. and Horton, K. (2014) Prevalence of Amyotrophic Lateral Sclerosis-United States, 2010-2011. MMWR. Surveillance Summaries, 63, 1-14.

[4] Wills, A., Hubbard, J., Macklin, E., Glass, J.,Tandan, R., Simpson, E., Brooks, B., Gelinas, D., Mitsumoto, H., Mozaffar, T., Hanes, G., Ladha, S., Heiman-Patterson, T., Katz, J., Lou, J.-S., Mahoney, K., Grasso, D., Lawson, R., Yu, H. and Cudkowicz, M. (2014) Hypercaloric Enteral Nutrition in Patients with Amyotrophic Lateral Sclerosis: A Randomised, Double-Blind, Placebo-Controlled Phase 2 Trial. The Lancet, 383, 2065-2072. http://dx.doi.org/10.1016/S0140-6736(14)60222-1

[5] Yin, H.Z., Nalbandian, A., Hsu, C.-I., Li, S., Llewellyn, K.J., Mozaffar, T., Kimonis, V.E. and Weiss, J.H. (2012) Slow Development of ALS-Like Spinal Cord Pathology in Mutant Valosin-Containing Protein Gene Knock-In Mice. Cell Death and Disease, 3, 1-12. http://dx.doi.org/10.1038/cddis.2012.115

[6] Purves, D. (2012) Neuroscience. 5th Edition, Sinauer Associates, Inc., Sunderland.

[7] Rothstein, J. (2009) Current Hypothesis for the Underlying Biology of Amyotrophic Lateral Sclerosis. Annals of Neurology, 65, S3-S9. http://dx.doi.org/10.1002/ana.21543

[8] Mitsumoto, H., Santella, R., Liu, X., Bogdanov, M., Zipprich, J., Wu, H.-C., Mahata, J., Kilty, M., Bednarz, K., Bell, D., Gordon, P.H., Hornig, M., Mehrazin, M., Naini, A., Beal, M.F. and Factor-Litvak, P. (2008) Oxidative Stress Biomarkers in Sporadic ALS. Amyotrophic Lateral Sclerosis, 9, 177-183. http://dx.doi.org/10.1080/17482960801933942

[9] Torbick, N., Hession, S., Stommel, E. and Caller, T. (2014) Mapping Amyotrophic Lateral Sclerosis Lake Risk Factors across Northern New England. International Journal of Health Geographics, 13, 1.

http://dx.doi.org/10.1186/1476-072X-13-1 
[10] Clark, J., Pritchard, C. and Sunak, S. (2005) Amyotrophic Lateral Sclerosis: A Report on the State of Research into the Cause, Cure, and Prevention of ALS. ALS Therapy Development Foundation, 1-15.

[11] Murata, T., Ohtsuka, C. and Terayama, Y. (2008) Increased Mitochondrial Oxidative Damage and Oxidative Damage DNA Damage Contributes to the Neurodegenerative Process in Sporadic Amyotrophic Lateral Sclerosis. Free Radical Research, 42, 221-225. http://dx.doi.org/10.1080/10715760701877262

[12] Robberecht, W. and Philips, T. (2013) The Changing Scene of Amyotrophic Lateral Sclerosis. Nature Reviews Neuroscience, 14, 248-264. http://dx.doi.org/10.1038/nrn3430

[13] Rodríguez-Hernández, Á., Cordero, M.D., Salviati, L., Artuch, R., Pineda, M., Briones, P., Sánchez-Alcázar, J.A., et al. (2009) Coenzyme Q Deficiency Triggers Mitochondria Degradation by Mitophagy. Autophagy, 5, 19-32. http://dx.doi.org/10.4161/auto.5.1.7174

[14] Rothstein, J. (1996) Excitotoxicity Hypothesis. Neurology, 47, 19S-26S. http://dx.doi.org/10.1212/WNL.47.4 Suppl 2.19S

[15] Choi, D.W. and Rothman, S.M. (1990) The Role of Glutamate Neurotoxicity in Hypoxic-Ischemic Neuronal Death. Annual Review of Neuroscience, 13, 171-182. http://dx.doi.org/10.1146/annurev.ne.13.030190.001131

[16] Keelan, J., Vergun, O. and Duchen, M.R. (1999) Excitotoxic Mitochondrial Depolarisation Requires Both Calcium and Nitric Oxide in Rat Hippocampal Neurons. The Journal of Physiology, 520, 797-813. http://dx.doi.org/10.1111/j.1469-7793.1999.00797.x

[17] Spreux-Varoquaux, O., Bensimon, G., Lacomblez, L., Salachas, F., Pradat, P.F., Le Forestier, N., Marouan, A., Dib, M. and Meininger, V. (2002) Glutamate Levels in Cerebrospinal Fluid in Amyotrophic Lateral Sclerosis: A Reappraisal Using a New HPLC Method with Coulometric Detection in a Large Cohort of Patients. Journal of the Neurological Sciences, 193, 73-78. http://dx.doi.org/10.1016/S0022-510X(01)00661-X

[18] Gordon, P.H. (2013) Amyotrophic Lateral Sclerosis: An Update for 2013 Clinical Features, Pathophysiology, Management and Therapeutic Trials. Aging and Disease, 4, 295-310. http://dx.doi.org/10.14336/AD.2013.0400295

[19] Aizawa, H., Sawada, J., Hideyama, T., Yamashita, T., Katayama, T., Hasebe, N., Kimura, T., Yahara, O. and Kwak, S. (2010) TDP-43 Pathology in Sporadic ALS Occurs in Motor Neurons Lacking the RNA Editing Enzyme ADAR2. Acta Neuropathologica, 120, 75-84. http://dx.doi.org/10.1007/s00401-010-0678-x

[20] Musaro, A. (2013) Understanding ALS: New Therapeutic Approaches. FEBS Journal, 280, 4315-4322. http://dx.doi.org/10.1111/febs.12087

[21] Wegorzewska, I., Bell, S., Cairns, N.J., Miller, T.M. and Baloh, R.H. (2009) TDP-43 Mutant Transgenic Mice Develop Features of ALS and Frontotemporal Lobar Degeneration. Proceedings of the National Academy of Sciences of the United States of America, 106, 18809-18814. http://dx.doi.org/10.1073/pnas.0908767106

[22] Yamashita, T., Chai, H.L., Teramoto, S., Tsuji, S., Kuniko, S., Muramatsu, S.I. and Kwak, S. (2013) Rescue of Amyotrophic Lateral Sclerosis Phenotype in a Mouse Model by Intravenous AAV9-ADAR2 Delivery to Motor Neurons. EMBO Molecular Medicine, 5, 1710-1719.

[23] Kabashi, E., Valdmanis, P.N., Dion, P., Spiegelman, D., McConkey, B.J., Velde, C.V., Bouchard, J.P., Lacomblez, L., Pochigaeva, K., Salachas, F., Pradat, P.F., Camu, W., Meininger, V., Dupre, N. and Rouleau, G.A. (2008) TARDBP Mutations in Individuals with Sporadic and Familial Amyotrophic Lateral Sclerosis. Nature Genetics, 40, 572-574. http://dx.doi.org/10.1038/ng.132

[24] Das, K., Nag, C. and Ghosh, M. (2012) Familial, Environmental, and Occupational Risk Factors in Development of Amyotrophic Lateral Sclerosis. North American Journal of Medical Sciences, 4, 350-355. http://dx.doi.org/10.4103/1947-2714.99517

[25] Bradley, W.G. and Mash, D.C. (2009) Beyond Guam: The Cyanobacteria/BMAA Hypothesis of the Cause of ALS and Other Neurodegenerative Diseases. Amyotrophic Lateral Sclerosis, 10, 7-20. http://dx.doi.org/10.3109/17482960903286009

[26] Bradley, W.G., Borenstein, A.R., Nelson, L.M., Codd, G.A., Rosen, B.H., Stommel, E.W. and Cox, P.A. (2013) Is Exposure to Cyanobacteria an Environmental Risk Factor for Amyotrophic Lateral Sclerosis and Other Neurodegenerative Diseases? Amyotrophic Lateral Sclerosis and Frontotemporal Degeneration, 14, 325-333. http://dx.doi.org/10.3109/21678421.2012.750364

[27] Morgan, R.H. and Srivastava, A.K. (2014) Clinical Relevance of Stem Cell Therapies in Amyotrophic Lateral Sclerosis. Neurology India, 62, 239-248. http://dx.doi.org/10.4103/0028-3886.136895

[28] Lamanauskas, N. and Nistri, A. (2008) Riluzole Blocks Persistent $\mathrm{Na}^{+}$and $\mathrm{Ca}^{2+}$ Currents and Modulates Release of Glutamate via Presynaptic NMDA Receptors on Neonatal Rat Hypoglossal Motorneurons in Vitro. European Journal of Neuroscience, 27, 2501-2514. http://dx.doi.org/10.1111/j.1460-9568.2008.06211.x

[29] Garbuzova-Davis, S., Sanberg, C.D., KuzminNichols, N., Willing, A.E., Gemma, C., Bickford, P.C., Miller, C., Rossi, R. and Sanberg, P.R. (2008) Human Umbilical Cord Blood Treatment in a Mouse Model of ALS: Optimization of Cell 
Dose. PLoS ONE, 3, e2494. http://dx.doi.org/10.1371/journal.pone.0002494

[30] Corti, S., Nizzardo, M., Nardini, M., Donadoni, C., Salani, S., Simone, C., Falcone, M., Riboldi, G., Govoni, A., Bresolin, N. and Comi, G.P. (2010) Systemic Transplantation of c-kit ${ }^{+}$Cells Exerts a Therapeutic Effect in a Model of Amyotrophic Lateral Sclerosis. Human Molecular Genetics, 19, 3782-3796. http://dx.doi.org/10.1093/hmg/ddq293

[31] Lepore, A.C., Rauck, B., Dejea, C., Pardo, A.C., Rao, M.S., Rothstein, J.D. and Maragakis, N.J. (2008) Focal Transplantation-Based Astrocyte Replacement Is Neuroprotective in a Model of Motor Neuron Disease. Nature Neuroscience, 11, 1294-1301. http://dx.doi.org/10.1038/nn.2210

[32] Ohnishi, S., Ito, H., Suzuki, Y., Adachi, Y., Wate, R., Zhang, J., Nakano, S., Kusaka, H. and Ikehara, S. (2009) IntraBone Marrow-Bone Marrow Transplantation Slows Disease Progression and Prolongs Survival in G93A Mutant SOD1 Transgenic Mice, an Animal Model Mouse for Amyotrophic Lateral Sclerosis. Brain Research, 1296, 216-224. http://dx.doi.org/10.1016/j.brainres.2009.08.012

[33] Lau, A. and Tymianski, M. (2010) Glutamate Receptors, Neurotoxicity and Neurodegeneration. European Journal of Physiology, 460, 525-542. http://dx.doi.org/10.1007/s00424-010-0809-1 